\title{
Ajustement d'un modèle hauteur-circonférence pour l'épicéa commun. Effet de la densité
}

\author{
C Deleuze 1,2, D Blaudez 2, JC Hervé 1,2 \\ 1 Laboratoire de biométrie, génétique et biologie des populations, CNRS-Ura 2055, \\ université Claude-Bernard Lyon-l, 43, bd du 11-Novembre-1918, 69622 Villeurbanne cedex; \\ 2 Unité dynamique des systèmes forestiers, Engref, 14, rue Girardet, 54042 Nancy cedex, France
}

(Reçu le 16 mai 1994 ; accepté le 2 mai 1995)

\begin{abstract}
Résumé - Un modèle hyperbolique à trois paramètres pour représenter les liaisons hauteur-circonférence est testé pour l'épicéa commun en peuplement équienne monospécifique. Les trois paramètres sont interprétables en termes de géométrie du nuage de points : le premier donne la valeur de l'asymptote, le second la pente à l'origine et le dernier est un paramètre de forme. Une procédure d'ajustement simultané est utilisée pour estimer globalement certains paramètres et étudier leur stabilité sur une famille de courbes (différentes densités, plusieurs années). Les données utilisées pour les ajustements proviennent d'un dispositif clinal et permettent de montrer qu'il n'y a pas d'effet significatif de la densité sur l'ajustement du modèle. L'effet de l'âge se traduit par une augmentation régulière de la pente à l'origine, tandis que l'asymptote suit l'évolution de la hauteur dominante et que le paramètre de forme reste stable. Le modèle hyperbolique est alors utilisé pour appréhender la compétition intraspécifique : un parallèle est fait entre l'épicéa, le chêne et le hêtre. Enfin, le modèle peut être aussi réduit en fixant le paramètre de forme à 0,9 et en effectuant un reparamétrage : il ne reste alors à ajuster chaque année que le paramètre de l'asymptote, elle-même très liée à la hauteur dominante.
\end{abstract}

relation hauteur-circonférence / densité / Picea abies / peuplement équienne monospécifique / compétition intraspécifique

Summary - Fitting a hyperbolic model for height versus girth relationship in spruce stands. Spacing effects. A hyperbolic model is used to represent height versus girth relationship in evenaged and pure stands of spruce. The three model parameters correspond to the geometric characteristics of the relationship: the asymptote, the slope at origin and the curve shape. A global fitting procedure is used for estimating some common parameters and for studying their stability in a range of stand density and age. The model is fitted with data from an experimental stand with a clinally varying density and it is not affected by spacing. The curve shape does not change with age, while the slope at origin and the asymptote increase. This model is used for comparing intraspecific competition in spruce, beech and oak stands. The shape parameter has little effect on adjustment and can be set to 0.9. The number of parameters can then be reduced to 1 by reparametrization: hence, only the asymptote is to be estimated, which is very close to top height.

height vs girth relationship / density / Picea abies / even-aged pure stand / intraspecific competition 


\section{INTRODUCTION}

L'étude de la dynamique des peuplements forestiers réguliers (monospécifiques équiennes) nécessite une meilleure compréhension à la fois de la croissance en circonférence et de celle en hauteur. Dans un peuplement fermé, où il existe des relations de compétition et une structure sociale, ces deux variables sont bien évidemment liées biologiquement : la hauteur assure un avantage dans la compétition pour la lumière, si bien que des arbres dominants auront une meilleure croissance et en particulier une plus forte circonférence. Cette compétition entretient ainsi une structure sociale dans le peuplement, véritable relation d'ordre entre les arbres. Il apparaît donc une liaison monotone entre la circonférence et la hauteur, à une date donnée. Cependant, les hauteurs ne suivent pas les mêmes variations que les circonférences : elles se stabilisent plus vite, les arbres dominants ayant des hauteurs voisines. Par conséquent, la liaison n'est pas linéaire et sa pente diminue avec la circonférence.

L'existence de cette liaison forte est largement utilisée en dendrométrie (appelée aussi "courbe des hauteurs" par les forestiers), puisqu'elle permet de calculer les hauteurs dominante et moyenne du peuplement à partir d'un échantillon restreint de mesures en hauteurs. En effet les mesures non destructives des hauteurs, à l'aide de dendromètres, sont longues et délicates : visée difficile dans un couvert fourni, imprécision de cette visée amplifiée par la hauteur de l'arbre (Pardé et Bouchon, 1988 ; Rai, 1979). L'ajustement d'une relation hauteur-circonférence sur un échantillon restreint du peuplement permet une estimation de la hauteur de tous les arbres, connaissant leur circonférence. De telles relations peuvent être utilisées pour calculer des volumes, pour compléter des modèles dendrométriques de croissance d'arbres (où la variable décrite est généralement la circonférence ou la surface ter- rière) ou pour analyser la structure du peuplement (Embry et Gottfried, 1971 ; Payandeh, 1984).

Mais le choix du modèle pour représenter ces relations n'est pas neutre. Parmi les modèles souvent utilisés, citons la droite, la parabole (Ker et Smith, 1955) ou les fonctions logarithmiques (Curtis, 1967 ; Wykoft et al, 1982). Ces modèles ont l'avantage d'être facilement ajustables avec des procédures de régression linéaire (Curtis, 1967 ; Farr et al, 1989), mais présentent en contrepartie un comportement divergent pour des valeurs extrêmes des circonférences. L'extrapolation de la relation s'avère alors délicate (Dolph, 1989), surtout si l'échantillon utilisé pour lajustement est réduit et déséquilibré. Le modèle hyperbolique de Dhôte et de Hercé (1994) semble plus robuste et mieux adapté à la géométrie du nuage de points hauteurs-circonférences (en particulier il possède une asymptote à l'infini). Ce modèle a été introduit par les auteurs pour deux feuillus (le hêtre et le chêne) ; nous nous proposons de le tester ici sur l'épicéa commun.

Par ailleurs, ces relations hauteur-circonférence évoluent avec l'âge du peuplement (en même temps que la hauteur dominante) (Bredenkamp, 1991), avec la fertilité de la station (Oswald, 1969 ; Nys et al, 1985 ; Bolstad et Allen, 1987 ; Larsen et Hann, 1987) ou avec la provenance génétique (Buford, 1986). L'impact du traitement sylvicole sur ces relations est plus sujet à polémique : rappelons que ces relations reflètent une certaine hiérarchie sociale issue de la compétition interarbres. De ce point de vue, une éclaircie réduisant la compétition doit agir aussi sur la relation. D'un autre point de vue, en séparant les deux variables, il est largement reconnu que la croissance en circonférence diminue avec la densité (Assmann, 1970 ; Seidel, 1984). Ceci a déjà pour effet de déplacer le nuage de points vers les circonférences les plus faibles. En revanche, la croissance en hauteur semble beaucoup moins sensible au 
traitement sylvicole (Seidel, 1984), au moins pour une large gamme de densités (Lanner (1985) parle même «d'axiome» pour les forestiers !). Si l'une des variables est défavorisée pendant que l'autre reste stable, qu'en sera-t-il de leur liaison? Remarquons que le modèle choisi pour représenter la liaison peut être une source de confusion : par exemple un modèle linéaire s'ajustera différemment suivant chaque densité pour tenir compte du déplacement du nuage de points vers les plus faibles circonférences. Avec le modèle hyperbolique pour le chêne et le hêtre, Dhôte et de Hercé ne trouvaient pas de différence significative suivant les densités.

Pour tester le modèle et l'effet de la densité, nous utiliserons un jeu de données provenant d'un dispositif clinal, avec un fort gradient de densité, sans variation de la station ni de la provenance génétique.

\section{MATÉRIEL ET MÉTHODES}

\section{Données}

\section{Dispositif expérimental}

Le dispositif clinal d'épicéa commun étudié (décrit par Dreyfus, 1990) est situé en forêt domaniale d'Amance, au nord-est de Nancy (Meurthe-etMoselle). Le dispositif a été mis en place par Décourt et Millier en 1970 (Décourt, 1970). Il est structuré à l'aide de deux gradients continus : du nord vers le sud, la densité évolue de la croissance libre (200 tiges/ha) vers une très forte densité (10 000 tiges/ha), sur $75 \mathrm{~m}$; de l'est vers l'ouest un mélange de deux provenances d'épicéas communs va d'un taux de $100 \%$ de Morzine à $100 \%$ d'Istebna, sur $50 \mathrm{~m}$ (fig 1). Mises à part ces contraintes de densité et de taux de mélange, les arbres sont répartis aléatoirement. Seuls les arbres de la provenance Istebna ont été mesurés dans la partie où cette provenance

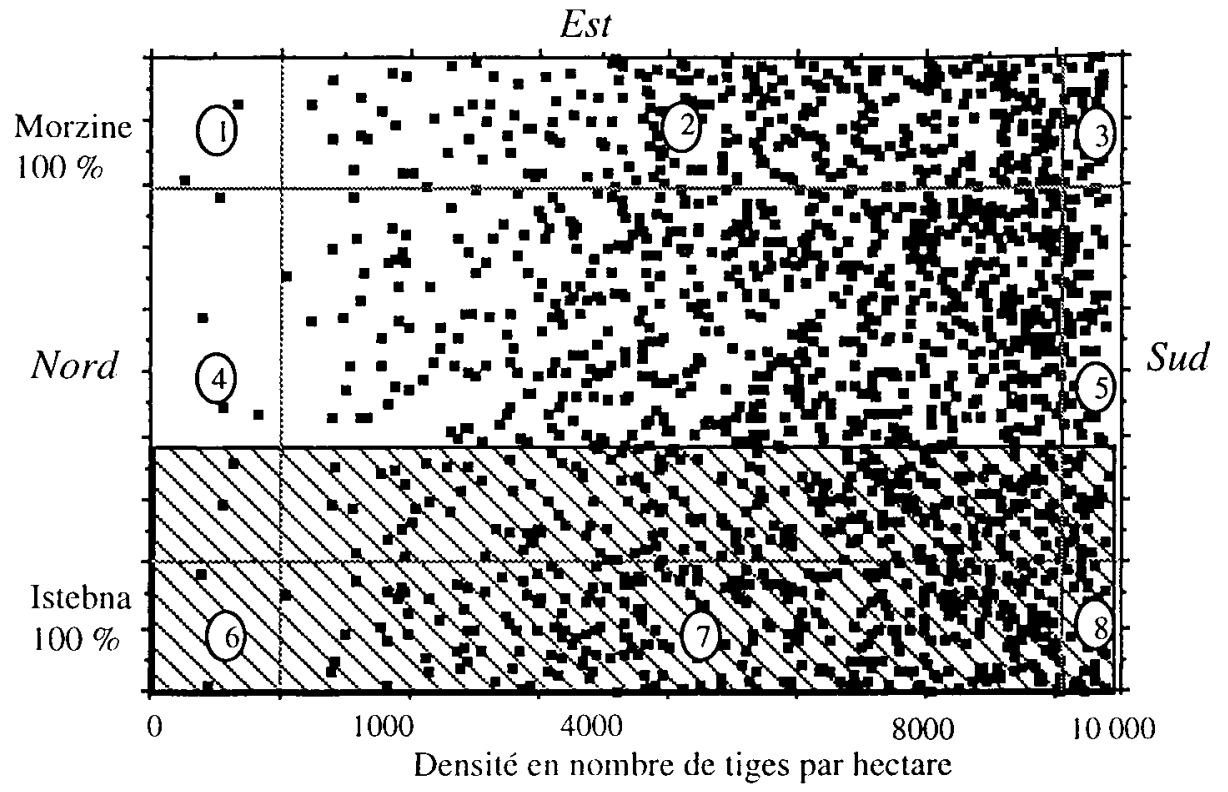

Fig 1. Plan du dispositif clinal d'Amance. Chaque point représente un arbre à la plantation. Sur chaque côté, une bande de $10 \mathrm{~m}$ sert de zone de protection pour la surface expérimentale centrale (placeau de $30 \times 60 \mathrm{~m}$ ), soient huit zones repérées sur ce plan. Seule la partie hachurée du dispositif a été utilisée pour la prise de données. 
constitue au moins deux tiers du mélange (zone hachurée sur la figure 1).

Environ 190 arbres (sur 375 de la zone hachurée) ont été mesurés pour cette étude, appartenant à tous les statuts sociaux dans les différentes densités. Les mesures ont été faites en hiver 1993, les arbres étaient âgés de 26 ans.

\section{Mesures des hauteurs}

Les arbres étaient abattus, ce qui a permis de repérer précisément leurs unités de croissance annuelle (délimitées par les cicatrices laissées par le bourgeon terminal, chaque hiver). Les hauteurs des premières années ont été recalées sur les mesures de hauteur totale faites sur pied, de 1970 à 1976 , ce qui enlève tout doute sur la datation des unités de croissance annuelle. La mesure sur arbres abattus (les arbres étant de surcroît assez rectilignes) évite les imprécisions usuelles des mesures des arbres sur pied faites avec des dendromètres à visée. D'autre part les arbres étant mesurés en une seule fois, l'origine est la même pour toutes les années (l'erreur est la même quelle que soit la hauteur).

\section{Mesures des circonférences}

Sur chaque arbre abattu, une rondelle a été prélevée à 1,30 m, pour mesurer les accroissements des cernes suivant deux diamètres perpendiculaires. Ces mesures permettent donc de reconstituer la croissance annuelle sous écorce (en moyenne, les épaisseurs d'écorce sont de 1,71士 0,02 mm, en 1993).

Finalement nous disposons donc de données appariées hauteur-circonférence sur une vingtaine d'années pour près de 200 arbres. L'originalité de ce jeu de données appariées est d'être relativement important $(50 \%$ des arbres de la bande étudiée), avec des mesures de hauteur assez fiables, des densités et des statuts sociaux contrastés, dans des conditions de croissance par ailleurs identiques (provenance génétique, station, années de croissance). II permet donc d'étudier l'effet de deux facteurs : l'âge (équivalent à l'année puisque tous les arbres ont le même âge) et la densité. Remarquons seulement que tous les statuts sociaux sont représentés à la date de mesure (échantillon équilibré en 1993). Cependant les arbres fortement dominés les premières années ont sans doute disparu si bien que l'échantillon est légèrement décalé vers les plus fortes circonférences les premières années (la sélection de la nature n'est pas aléatoire !). Ce défaut est cependant peu gênant pour cette étude : d'une part la période de temps est courte (moins de 20 ans), donc peu d'arbres sont concernés, et d'autre part le peuplement est encore peu structuré pendant les premières années (avant la fermeture du couvert), si bien que le modèle n'est utilisé dans un premier temps qu'à partir de l'année 1976.

\section{Logiciel Multilisa d'ajustements non-linéaires}

Tous les ajustements présentés ici ont été réalisés à l'aide du logiciel Multilisa développé sur Sun, par JC Hervé. Multilisa est un logiciel d'ajustement non-linéaire qui utilise un algorithme du type Gauss-Marquardt sur un critère des moindres carrés ordinaires. II permet d'ajuster un modèle à des familles de courbes, en prenant éventuellement des paramètres communs pour la famille (ajustement «global» du paramètre).

\section{Présentation du modèle}

\section{Équation du modèle (Dhôte et de Hercé, 1994)}

Le modèle initial correspond au modèle hyperbolique de Dhôte et de Hercé, utilisé pour le hêtre et le chêne. Il donne la hauteur $(H$ en $\mathrm{m})$ en fonction de la circonférence à $1,30 \mathrm{~m}(C$ en $\mathrm{cm})$ :

$H(C)=\frac{\left(\mu_{1}+\mu_{2} C-\sqrt{\left.\left(\mu_{1}+\mu_{2} C\right)^{2}-4 \mu_{1} \mu_{2} \mu_{3} C\right)}\right.}{2 \mu_{3}}+1,3$

où $\mu_{1}$ (en $\mathrm{m}$ ) correspond à l'asymptote pour les fortes valeurs de $C, \mu_{2}(\mathrm{~m} / \mathrm{cm})$ est la pente à l'origine et $\mu_{3}$ (sans unité), un paramètre de forme (quand $\mu_{3}$ tend vers 1 , l'hyperbole tend vers deux segments de droite).

Ce modèle présente les avantages suivants : i) c'est une courbe croissante positive sur $[0,+\infty[$; ii) il passe par le point $H=1,30 \mathrm{~m}, C=0$; iii) il possède une asymptote pour les plus fortes circonférences, qui est en pratique liée à la hau- 
teur dominante du peuplement ; iv) dans le cas du hêtre ou du chêne, deux paramètres sur les trois semblent très stables pour un même peuplement, ce qui permet d'ajuster des faisceaux de courbes, où le seul paramètre estimé $\left(\mu_{1}\right)$ est lié à la hauteur dominante.

Ce modèle rend compte de la structuration sociale du peuplement : des arbres dominants avec les plus fortes circonférences et une croissance en hauteur peu liée à la circonférence (palier de l'asymptote), et des arbres dominés avec une croissance en circonférence très ralentie et une croissance en hauteur faible (liaison linéaire au départ).

\section{Fonctions de sensibilité}

Les trois fonctions de sensibilité du modèle, c'està-dire les dérivées par rapport aux paramètres, sont tracées à titre d'exemple pour les paramètres trouvés l'année 1992. Elles renseignent sur les zones où le modèle sera le plus sensible aux variations des paramètres, donc où il faudra le plus de données pour estimer au mieux le paramètre. Afin de tracer sur un même graphique (fig 2) les trois fonctions de sensibilité et le modèle, nous avons utilisé un paramètre d'échelle (20 pour le modèle, 1 pour la dérivée par rapport à $\mu_{1}$, 16 pour celle par rapport à $\mu_{2}$ et 1600 pour $\mu_{3}$ ). Les deux premières fonctions de sensibilité montrent qu'il est nécessaire d'avoir des données bien réparties tout le long de la courbure de l'hyperbole : en début pour le paramètre $\mu_{2}$ qui donne la pente à l'origine et à la fin pour l'asymptote $\mu_{1}$ (c'est relativement logique). Même si le modèle hyperbolique a un comportement cohérent avec les relations hauteur-circonférence (en

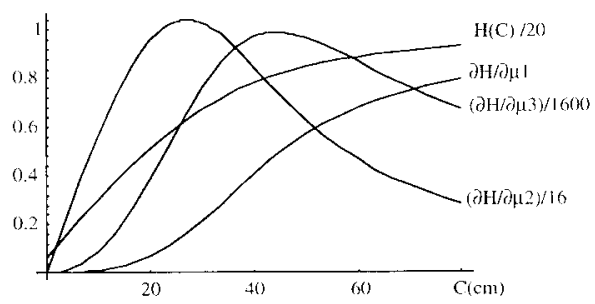

Fig 2. Fonction hyperbolique et les trois fonctions de sensibilité, correspondant à chacun des 3 paramètres de $1992\left(\mu_{1}=18,872 ; \mu_{2}=\right.$ 0,$00494 ; \mu_{3}=0,88$ ) (en abscisse : circonférences en $\mathrm{cm}$ ). particulier l'asymptote à l'infini), ces fonctions de sensibilité montrent que ce modèle ne peut pallier une mauvaise répartition des données. Enfin, la fonction de sensibilité de $\mu_{3}$ est presque proportionnelle à celle de $\mu_{2}$ au début de la courbe et plus encore à celle de $\mu_{1}$ sur les deux tiers de la courbe : ceci indique que, pour séparer l'effet du paramètre $\mu_{3}$ des deux autres, il faut des mesures des plus gros arbres (au-delà de $50 \mathrm{~cm}$ de circonférence à 26 ans, sur notre exemple).

\section{Qualité de l'ajustement}

La qualité des ajustements est mesurée par la somme des carrés des écarts (notée $\Sigma 2$ ) pour ces ajustements par moindres carrés. Cette somme permet d'évaluer le gain entre deux modèles emboîtés : M1 $\subset$ M2 (soit pour le nombre de paramètres : $p 2>p 1$ ), grâce au rapport :

$$
\frac{\left(\sum 2(1)-\sum 2(2)\right) /(p 2-p 1)}{\sum 2(2) /(n-p 2)}
$$

( $n$ est le nombre d'observations). Ce rapport suit asymptotiquement une loi de Fisher $F(p 2-p 1, n-$ p2) (si le modèle le plus simple (M1) est correct, il est accepté quand le rapport est inférieur à la valeur du $F$, ou quand la probabilité associée est supérieure à $5 \%$ ).

\section{Prise en compte des effets âge et densité}

L'influence de l'âge et de la densité sur la relation hauteur-circonférence est appréciée par l'intermédiaire des variations des différents paramètres du modèles $H(C)$ en fonction de ces deux facteurs. Bien qu'il s'agisse de deux facteurs quantitatifs, ils ont été traités comme qualitatifs, pour éviter d'avoir à formuler des hypothèses sur la forme de leur éventuelle liaison avec les paramètres. L'âge et la densité constituent ainsi deux facteurs croisés fixes.

En réalité, les mêmes arbres ayant été mesurés à différents âges, il y aurait un troisième facteur à prendre en compte, le facteur arbre: il s'agirait d'un facteur aléatoire, croisé avec l'âge et subordonné à la densité. La prise en compte de ce troisième facteur conduirait à un modèle non linéaire (le modèle $H(C)$ ) à effets mixtes. Mais l'ajustement d'un tel modèle n'est pas élémen- 
taire (et d'ailleurs pas nécessairement réalisable dans le cas présent). Dans un souci de simplicité, nous avons préféré ne pas tenir compte de l'effet arbre. Dans ces conditions, l'étude simultanée des effets âge et densité peut poser problème : négliger l'effet arbre risque d'amener à conclure beaucoup trop facilement à un effet densité (par confusion avec l'effet arbre), et ceci d'autant plus que le nombre de modalités du facteur âge sera grand, ce qui est le cas ici. Pour éviter cela, nous avons adopté la démarche suivante. Dans un premier temps, nous avons étudié l'effet densité âge par âge (le problème de l'effet arbre ne se pose alors pas, puisqu'un même arbre n'est mesuré qu'une fois à un âge donné). L'effet densité âge par âge se révélant non significatif, nous avons étudié, dans un deuxième temps, l'effet âge toutes densités confondues, en négligeant toujours l'effet arbre. Les estimations années par années sont alors correctes, mais non indépendantes : la conséquence est de conclure trop facilement à l'absence d'effet âge.

Dans l'ensemble, les deux temps de la procédure préservent (pour des raisons différentes) le niveau du risque de première espèce (conclure à des effets qui n'existent pas), au dépend du risque de deuxième espèce (ne pas déceler des effets qui existent).

\section{RÉSULTATS ET DISCUSSION}

\section{Effet de la densité sur la relation $\mathrm{H}=\mathrm{f}(\mathrm{C})$}

En travaillant année par année (à partir de 1980), les données ont été séparées par classe de densité de 1000 en 1000 tiges/ha (la densité évoluant de 200 à 10000 tha, on a neuf classes de densité (de N2 à N10) dans le placeau, avec une vingtaine d'arbres par densité, une classe (N1) de "croissance libre" dans la zone de protection nord et une classe (N11) à 10000 tiges/ha, pour la zone de protection sud. Pour la classe N1 il n'y a pas assez de données et elle n'est pas prise en compte dans les ajustements par classe).

L'ajustement avec tous les paramètres locaux (soit trois paramètres par classe de densité) n'est pas possible car il y a trop peu de données par classe (de 10 à 30 suivant les densités). Dans la mesure où le paramètre de forme a une faible influence sur l'ajustement du modèle nous avons choisi d'ajuster globalement le paramètre $\mu_{3}$ (c'est- à-dire simultanément pour toutes les densités de l'année), sauf dans un cas où $\mu_{1}$ et $\mu_{2}$ sont ajustés globalement, et $\mu_{3}$ localement, pour tester la sensibilité du paramètre de forme à la densité. Finalement, les différents modèles sont:

- un modèle «local» par densité , c'est-àdire où $\mu_{1}$ et $\mu_{2}$ sont ajustés pour chaque classe de densité (soit $2 \times 10+1$ paramètres : modèle $\mathrm{m} 5$ du tableau I) ;

- trois modèles avec l'un des paramètres ajusté localement tandis que les deux autres sont globaux (étude de la sensibilité de ce paramètre à la densité, avec $10+2$ paramètres) (modèles $\mathrm{m} 2, \mathrm{~m} 3$ et $\mathrm{m} 4 \mathrm{du}$ tableau I) ;

- enfin, un modèle "global» où tous les paramètres sont communs aux différentes densités ( 3 paramètres) (modèle $\mathrm{m} 1 \mathrm{du}$ tableau I) ;

Nous avons comparé ces modèles emboîtés, à l'aide du test $F$ (à titre d'exemple les résultats des années 1985 et 1990 sont donnés dans le tableau I, les résultats étant qualitativement les mêmes pour les autres années).

Statistiquement on ne décèle pas de différences entre les modèles, le modèle le plus simple (les trois paramètres globaux) est ainsi retenu et il n'y a pas lieu de distinguer les différentes densités. Graphiquement l'ajustement sur 10 classes de densité avec 21 paramètres aboutit à un faisceau croisé de courbes. La représentation graphique des paramètres en fonction de la densité (fig 3) ne fait pas apparaître de tendance. Les différences entre ajustements sont plus à attribuer à l'incertitude autour des paramètres et à la répartition légèrement différente des données par den- 
Relation hauteur-circonférence pour l'épicéa

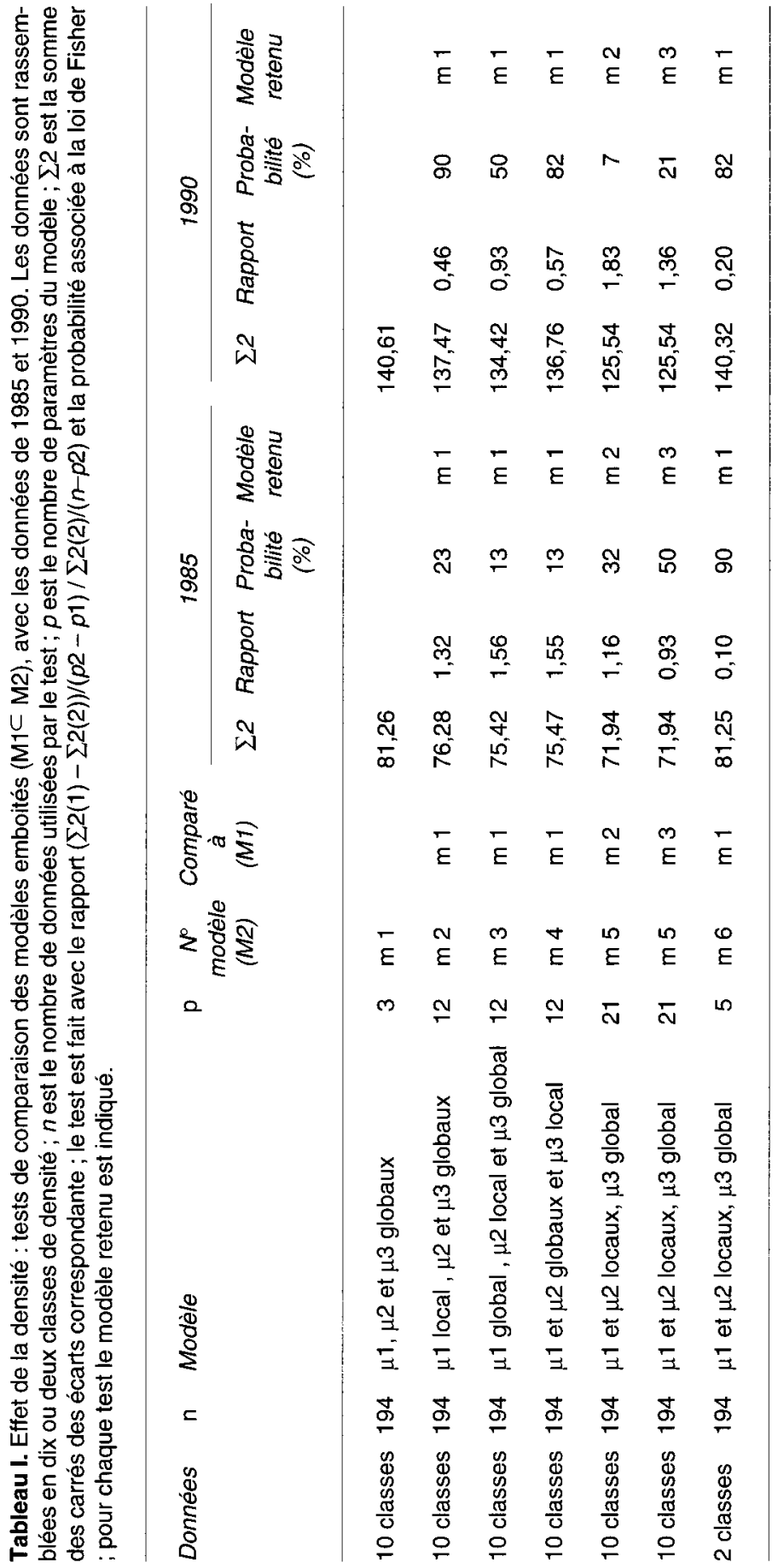


sité (à faible densité on trouve plus de points autour de l'asymptote et en forte densité au contraire plus de points en début de courbe). Pour augmenter le nombre de données par densité et améliorer la qualité des ajustements, seules deux classes de densité sont distinguées (les densités très fortes au-delà de 5000 t/ha et les densités faibles ou

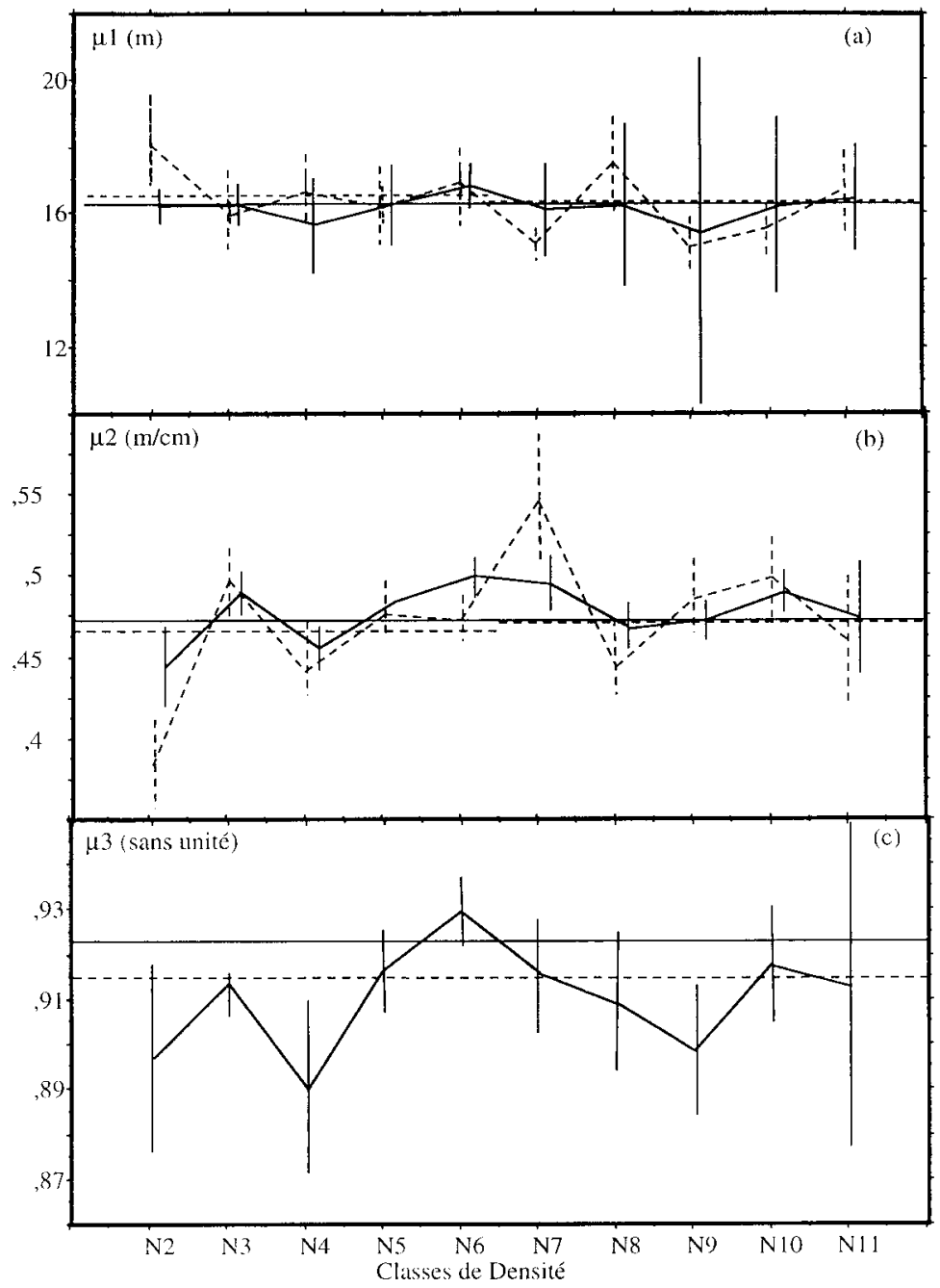

Fig 3. Paramètres $\mu_{1}, \mu_{2}$ et $\mu_{3}$ en fonction des classes de densité, pour l'année 1990, suivant différents modèles sur les données rassem. blées en dix ou deux classes de densité. Les barres verticales représentent les écarts types. 
moyennes en deçà de $5000 \mathrm{t} / \mathrm{ha}$ ). Cette fois le modèle le plus complet a cinq paramètres $\left(\mu_{1}\right.$ et $\mu_{2}$ locaux, et $\mu_{3}$ global : modèle $\mathrm{m} 6 \mathrm{du}$ tableau I). Les résultats statistiques (tableau I) sont encore plus en faveur d'un modèle unique quelle que soit la densité.

Finalement, avec le modèle hyperbolique, on ne peut pas mettre en évidence d'effet de la densité sur la relation hauteur-circonférence. Remarquons que, sur notre jeu de données, l'ajustement d'un autre modèle (droite ou parabole par exemple) donnerait des résultats différents par densité (du fait du décalage des points). Ce résultat a un impact pratique puisqu'il permet de considérer les données globalement toutes densités confondues.

Cependant, cela ne veut pas dire que la densité n'a pas d'effet sur le nuage de points hauteur-circonférence. Au contraire nous avons constaté que la densité a un impact sur la position de ce nuage. Même si ces points se trouvent toujours plus ou moins sur la courbe hyperbolique, ils sont décalés vers les fortes circonférences en faible densité, et inversement pour les fortes densités (influence directe de la densité sur la croissance en circonférence). Si la distribution des points n'est pas la même, les arbres les plus gros se retrouvent tout de même toujours autour de l'asymptote, c'està-dire à un même niveau de hauteur. En particulier la hauteur dominante (hauteur moyenne des 100 plus gros arbres à l'hectare) est stable quelle que soit la densité, et est très liée à la valeur de $\mu_{1}$. À la limite, en très forte densité, le nuage de points sera décalé en tout début d'asymptote ce qui pourra induire une légère diminution de la hauteur dominante : ceci correspond à l'effet inhibiteur de très fortes densités sur la croissance en hauteur.

Ce modèle semble donc assez cohérent avec les résultats classiques sur l'impact de la densité sur la croissance du peuplement (en circonférence et en hauteur).
D'autre part l'asymptote du modèle semble encore moins sensible à la densité que la hauteur dominante.

\section{Évolution des paramètres avec l'âge du peuplement}

\section{Modèles emboîtés : choix du modèle optimal}

L'étude de l'effet de la densité justifie que l'on fasse des ajustements toutes densités confondues. Il est alors possible d'étudier l'évolution des paramètres avec l'âge du peuplement (sur 17 ans, l'ajustement n'étant possible qu'à partir de 1976). À nouveau on teste plusieurs modèles emboîtés :

- un ajustement local, c'est à dire indépendamment pour chaque année (trois paramètres par année, soit 51 paramètres : modèle $\mathrm{m} 1 \mathrm{du}$ tableau II) ;

- un ajustement local des paramètres $\mu_{1}$ et $\mu_{2}$ en prenant le paramètre $\mu_{3}$ commun pour toutes les années (soit $2 \times 17+1$ paramètres : modèle $\mathrm{m} 2$ du tableau II) ;

- le paramètre $\mu_{1}$ local et les deux autres $\left(\mu_{2}\right.$ et $\left.\mu_{3}\right)$ communs pour toutes les années $(17+2$ paramètres : modèle $m 3$ du tableau II).

$\mu_{1}$ représentant l'asymptote ne peut évidemment pas être commun pour toutes les années.

Ces différents modèles sont comparés dans le tableau II à l'aide du test $F$. Rappelons que ce test, ne tenant pas compte de l'effet arbre, a tendance à favoriser les modèles globaux. D'après le tableau II, nous retiendrons cependant le modèle avec $\mu_{3}$ commun pour toutes les années (m2). De toute façon, le modèle hyperbolique est peu sensible à une petite variation du paramètre de forme. Les graphes bivariés des paramètres et de leurs ellipses de confiance à $50 \%$ (fig 4) appuient ce choix : dans le modèle le plus complet (fig $4 a, b$ ), les 


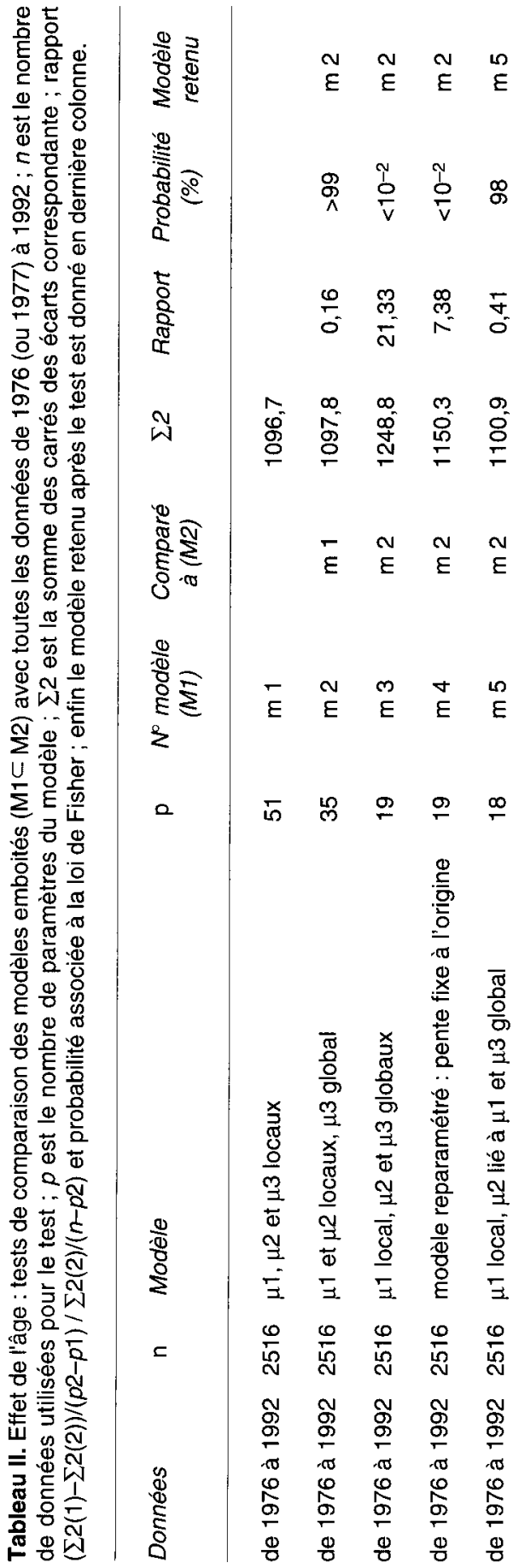




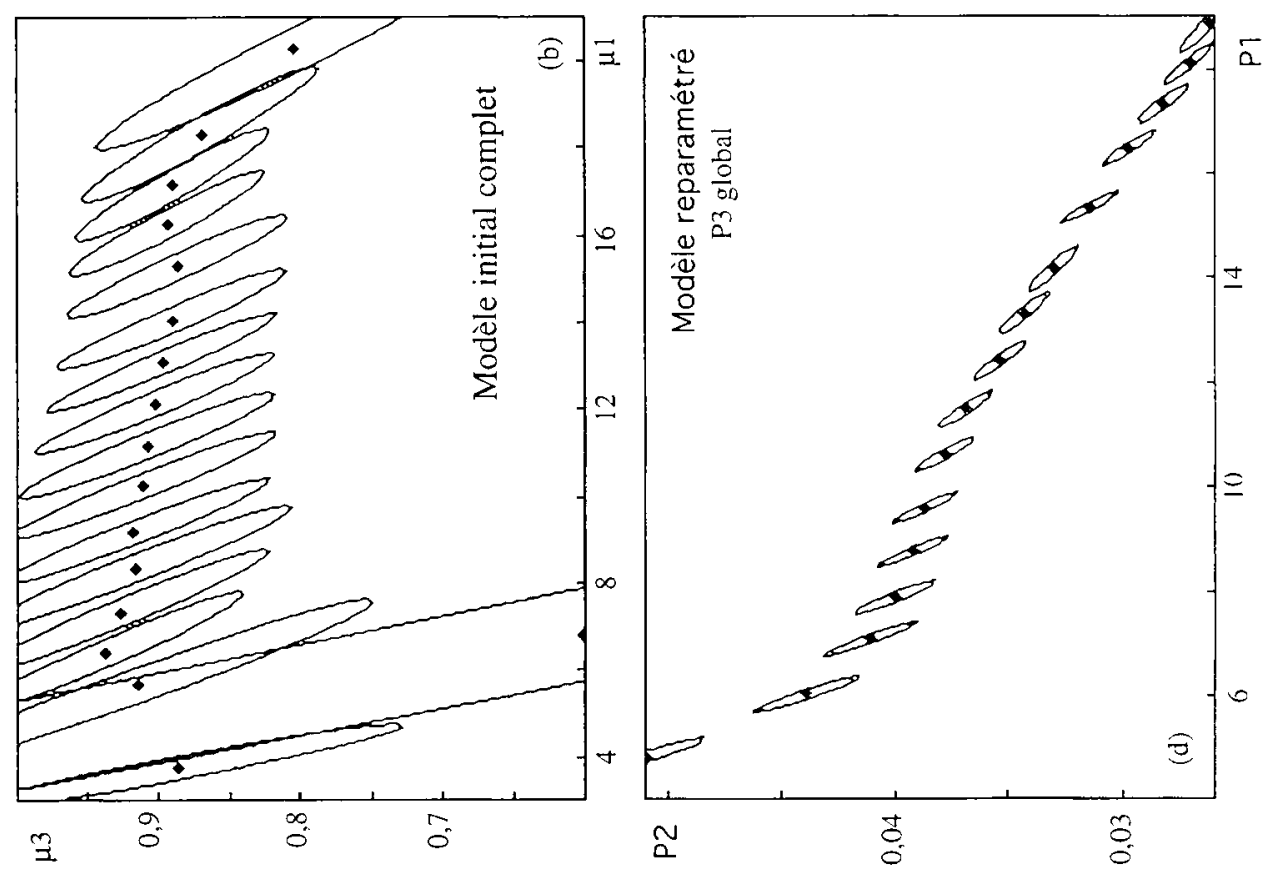

守

을

ह $\frac{0}{\mathrm{~N}}$

$\Phi 0$

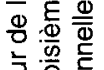

느을 을

范苛

草

Ф

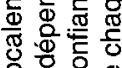

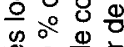

용응

尚必然

용 응 क

需

텅 응

क

为造

心

옹 웡원

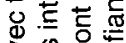

元禺

幽

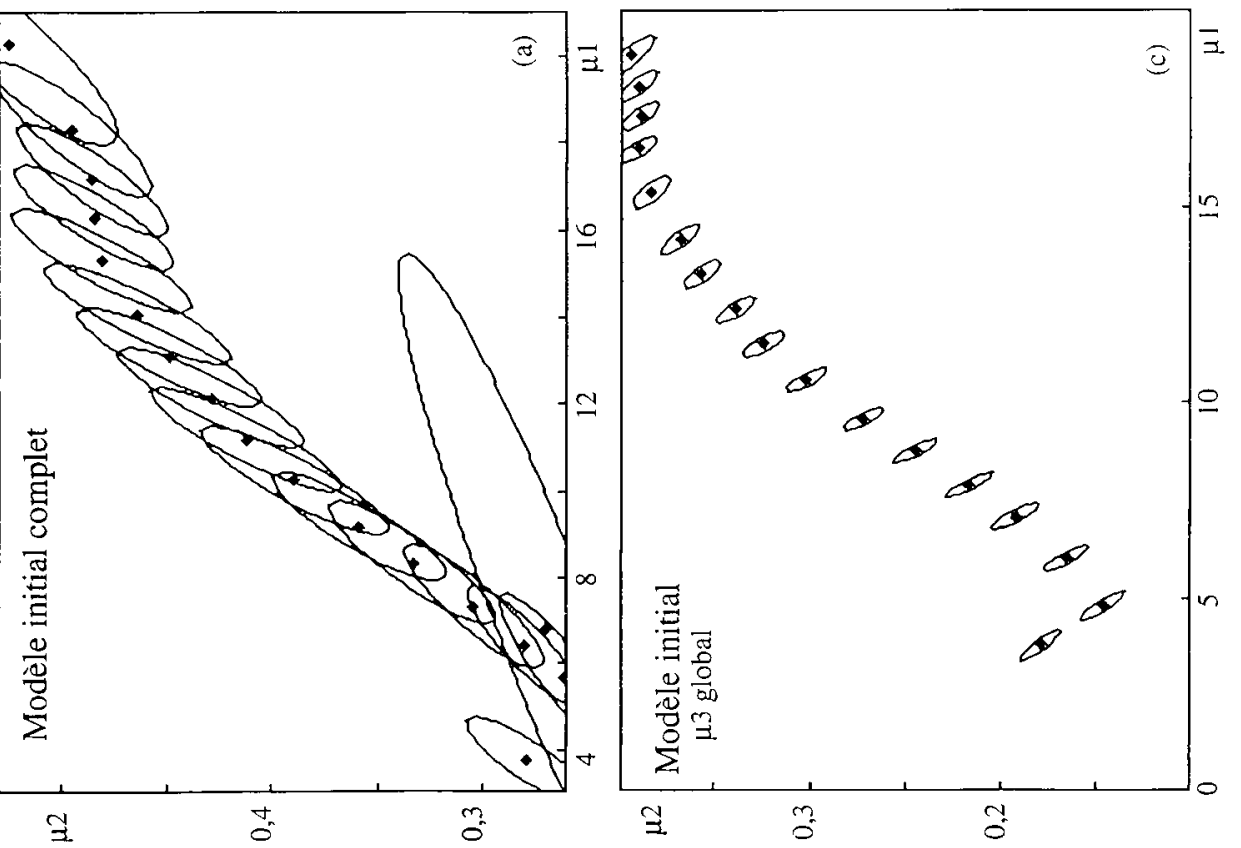

을 음 임

엉 등

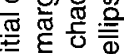

웡

응 는

은 은

등 총 눙

눙웡

๘

in 응응

¿

홍음

팅

历.

造

号总产

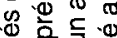

흔 홍 똔

空

क

怘芒元

준

○

긍ㅎㅇ

읜 
ellipses sont très larges (notamment suivant $\mu_{3}$ ) et inclinées, ce qui indique à la fois une grande imprécision sur les estimations et une forte corrélation entre les paramètres. Au contraire, elles sont étroites dans le modèle avec $\mu_{3}$ commun (fig 4c). Le graphe bivarié indique une forte relation non linéaire entre $\mu_{1}$ et $\mu_{2}$, mais les ellipses de confiance ne sont pas inclinées dans le sens de cette liaison, ce qui ne remet pas en cause la qualité de l'ajustement. De plus, la répartition des résidus autour de l'axe des abscisses (fig $5 a$ ) indique qu'il n'y a pas de biais.

D'autre part, l'ajustement se révèle peu sensible aux variations de $\mu_{3}$ et ce paramètre peut être fixé en pratique à une valeur arbitraire de 0,9 (la valeur de l'ajustement global de $\mu_{3}$ est $0,882 \pm 0,0016$ ). Le modèle a alors un paramètre en moins avec une somme des carrés (modèle avec $\mu_{3}$ fixé : $\left.\Sigma^{2}=1098,39\right)$ très voisine de celle du modèle retenu (modèle avec $\mu_{3}$ global : $\Sigma^{2}=$ $1097,86)$.

Enfin, le modèle avec un seul paramètre local $\left(\mu_{1}\right)$ est rejeté, ce qui traduit le fait que la pente à l'origine ne peut être commune à toutes les courbes : elle augmente avec l'asymptote (fig 6).

\section{Reparamétrages du modèle}

Afin de réduire le nombre de paramètres locaux, on teste un modèle reparamétré où la pente à l'origine peut éventuellement suivre les variations de l'asymptote: $P_{1}=\mu_{1}$, $P_{2}=\mu_{2} / \mu_{1}$ et $P_{3}=\mu_{3}$, soit :

$H(C)=P_{1}\left\{\frac{1+P_{2} C-\sqrt{\left(\left(1+P_{2} C\right)^{2}-4 P_{2} P_{3} C\right)}}{2 P_{U}}+1,3\right\}$

L'ajustement de ce modèle reparamétré en prenant $P_{3}$ global, avec $P_{1}$ et $P_{2}$ locaux donne exactement les mêmes résultats que pour le modèle initial, mais cette fois l'ajustement global de $P_{2}$ (modèle à $17+2$ paramètres : modèle $\mathrm{m} 4 \mathrm{du}$ tableau II) autorise l'augmentation de la pente à l'origine avec l'asymptote. Cependant le test $F$ nous fait rejeter ce dernier modèle, d'autant plus que ce test est à nouveau trop optimiste pour le modèle global. Enfin, les résidus correspondants à ce modèle (fig $5 \mathrm{~b}$ ) ont tendance à augmenter avec la circonférence, ce qui montre à nouveau que le modèle est à rejeter.

Le graphe bivarié (fig $4 \mathrm{~d}$ ) de $P_{2}$ en fonction de $P_{1}$ montre une forte liaison linéaire entre les deux paramètres $\left(P_{2}=0,0543-\right.$ $0,0015{ }^{*} P_{1}$ ). Le modèle est reparamétré avec cette relation, ce qui réduit encore le nombre de paramètres (seul $P_{1}$ est encore ajusté et $P_{3}$ est global, donc il ne reste plus que $17+1$ paramètres). Cette fois le test $F$ (modèle $\mathrm{m} 5$, tableau II) est fortement en faveur de ce modèle plus simple à un seul paramètre par année $(p=98 \%)$. Les résidus (fig $5 c$ ) ne présentent pas de tendance particulière. Ce modèle atteint alors le même niveau de réduction que celui de Dhôte et de Hercé, mais dans le cas de l'épicéa la pente à l'origine augmente avec l'asymptote. Comme le remarquent les auteurs, l'introduction de fortes contraintes sur le modèle permet de traiter des échantillons plus réduits ou déséquilibrés (avec surtout de gros arbres pour estimer correctement $P_{1}$ ). Cependant il convient de préciser quelques limites à ce reparamétrage, même si le rapport de vraisemblance est en faveur de ce modèle. En effet, il est contingent au premier ajustement de $P_{1}$ et $P_{2}$ pour déterminer la relation linéaire entre les deux paramètres : en particulier avec l'étude du seul dispositif clinal, il ne peut être généralisé à d'autres peuplements, et il n'a été testé que sur les 20 premières années, sans information sur la relation entre $P_{1}$ et $P_{2}$ à plus long terme. Remarquons que Ker et Smith (1955) atteignaient le même niveau de réduction pour leur modèle parabolique, avec une hauteur maximale (correspondant à la hauteur dominante, donc à notre paramètre $P_{1}$ ) et une relation entre cette hau- 

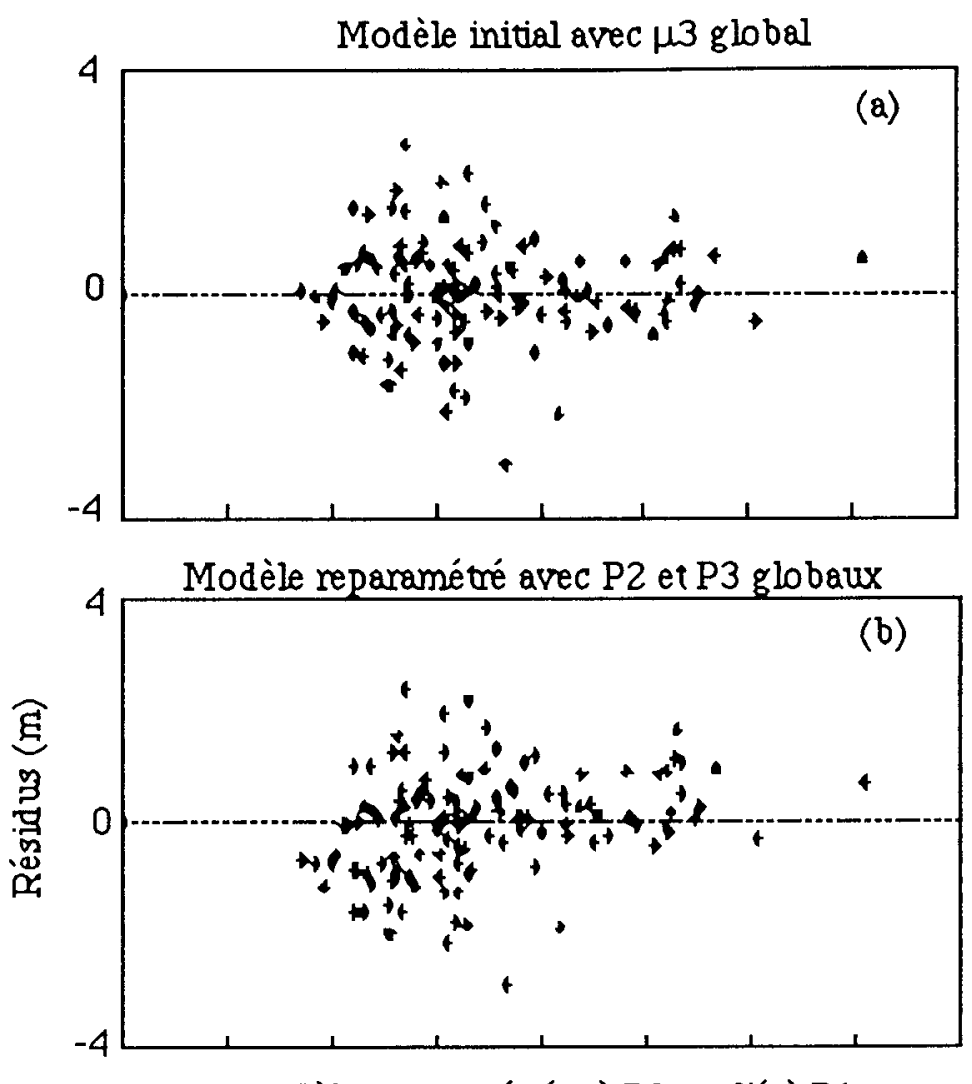

Fig 5. Graphes des résidus pour les données de 1992 : a modèle avec $\mu_{3}$ commun pour toutes les années ; $\mathbf{b}$ modèle reparamétré avec $P_{2}$ et $P_{3}$ globaux ; $c$ modèle reparamétré avec $P_{2}$ lié à $P_{1}$ et $P_{3}$ ajusté globalement.
Modèle reparamétré où P2 est lié à P1

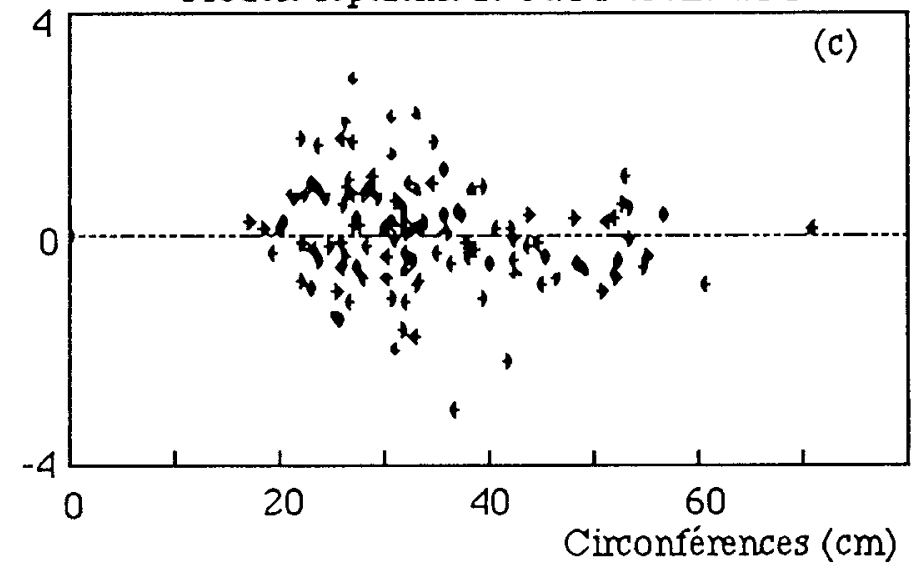




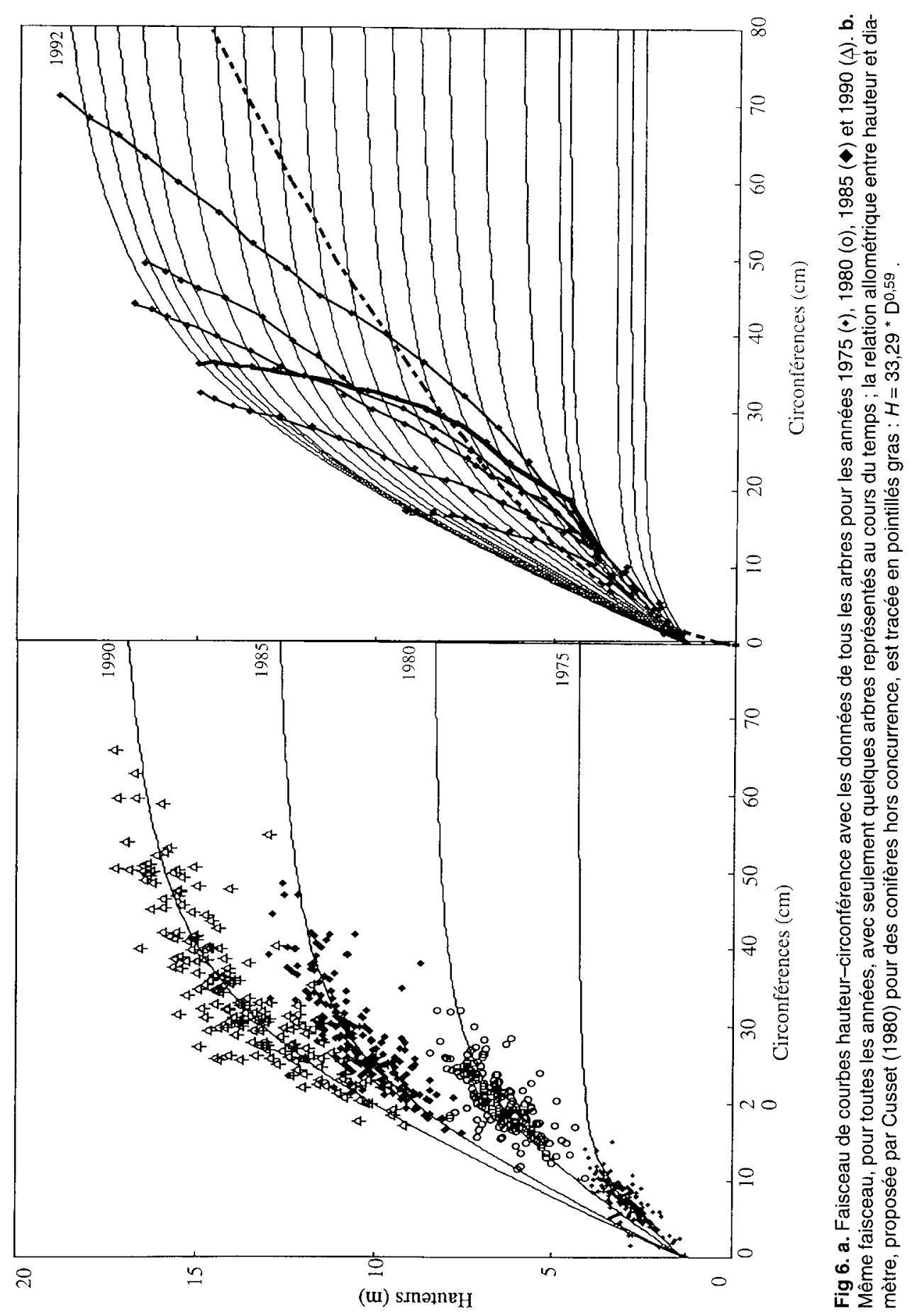


teur et la circonférence maximale (notre liaison entre $P_{1}$ et $P_{2}$ ). Finalement le premier modèle $\left(\mu_{1}, \mu_{2}\right.$ locaux et $\mu_{3}$ global) est préférable a priori puisqu'il reste indépendant du jeu de données. Une reparamétrisation sera possible, par exemple pour pallier un manque de données. Mais ce modèle reparamétré sera à utiliser avec prudence, en étant conscient des risques de biais et d'extrapolation à plus long terme ou sur d'autres peuplements.

Rappelons que l'ajustement du modèle le plus complet n'est possible qu'à partir de l'année 1976, mais la réduction du modèle (par exemple en prenant $\mu_{3}$ global) permet d'ajuster le modèle sur les premières années (pour un trop jeune peuplement il ne se dégage pas une structure assez forte du nuage de points hauteur-circonférence pour estimer un paramètre de forme). Cet ajustement permet par exemple d'obtenir un faisceau de courbes sur toutes les années (fig 6).

\section{Évolution des paramètres avec l'âge}

Sur la figure 7, nous n'avons représenté que les valeurs des paramètres des modèles retenus après un test $F$ (modèle initial avec $\mu_{3}$ ajusté globalement ou fixé à 0,9 et le modèle reparamétré avec $P_{2}$ lié à $P_{1}$ ) ainsi que le modèle le plus complet avec tous ses paramètres locaux. D'autre part, la hauteur dominante (hauteur moyenne des 100 plus gros arbres par hectare) est représentée avec le graphe du paramètre $\mu_{1}$. Cette hauteur dominante est soit calculée à partir des données (hauteur moyenne des plus gros arbres), soit estimée à partir des modèles et de la circonférence dominante $(f(C o))$.

Sur la figure 7a, l'évolution de l'asymptote est très voisine pour tous les modèles et très proche de la hauteur dominante (calculée directement ou à partir des modèles), surtout pour le modèle retenu avec $\mu_{3}$ global. II semble que sur ces premières années le paramètre $\mu_{1}$ soit un bon indicateur de la hauteur dominante, ce que constataient Dhôte et de Hercé pour leurs peuplements de chênes et de hêtres. L'écart type sur l'asymptote pour le modèle retenu est au maximum (en 1992) de $0,3 \mathrm{~m}$ (avec $\mu_{1}=$ $18,28 \mathrm{~m}$ ).

Sur la figure $7 b$ et $c$, les intervalles de confiance (représentés seulement pour $\mu_{2}$ et $\mu_{3}$, pour la lisibilité du graphe de $\mu_{1}$ ) se réduisent énormément en améliorant l'ajustement, et les courbes des paramètres sont plus lisses et régulières.

\section{SUIVI DE QUELQUES ARBRES}

Il est intéressant de suivre quelques arbres pris arbitrairement dans différentes classes sociales (fig $6 \mathrm{~b}$ ) : à droite les arbres de l'étage dominant et codominant, avec des hauteurs au niveau de l'asymptote, et une croissance régulière chaque année en hauteur et en circonférence. À gauche les arbres plutôt dominés ont tendance à «favoriser» leur croissance en hauteur (pour maintenir leur houppier dans la canopée) au détriment de la croissance en circonférence (quasiment nulle pour l'arbre le plus dominé). La plupart du temps ces courbes ne se coupent pas (la «relation d'ordre» entre les arbres est conservée, et la croissance en circonférence est plus vite ralentie que la croissance en hauteur). Cependant il arrive qu'un arbre de l'étage dominant ralentisse subitement sa croissance en circonférence (exemple en gras) et se retrouve dans l'étage dominé (sans doute à cause d'un accident physiologique ou physique : des cas d'attaque de Fomes annonus sont signalés dans le dispositif). Globalement ce modèle rend ainsi bien compte de la structure sociale du peuplement, et c'est sans doute pour cela qu'il s'ajuste moins bien dans les premières années, quand la structuration est encore faible. De même nous ne l'avons pas réellement testé pour des 


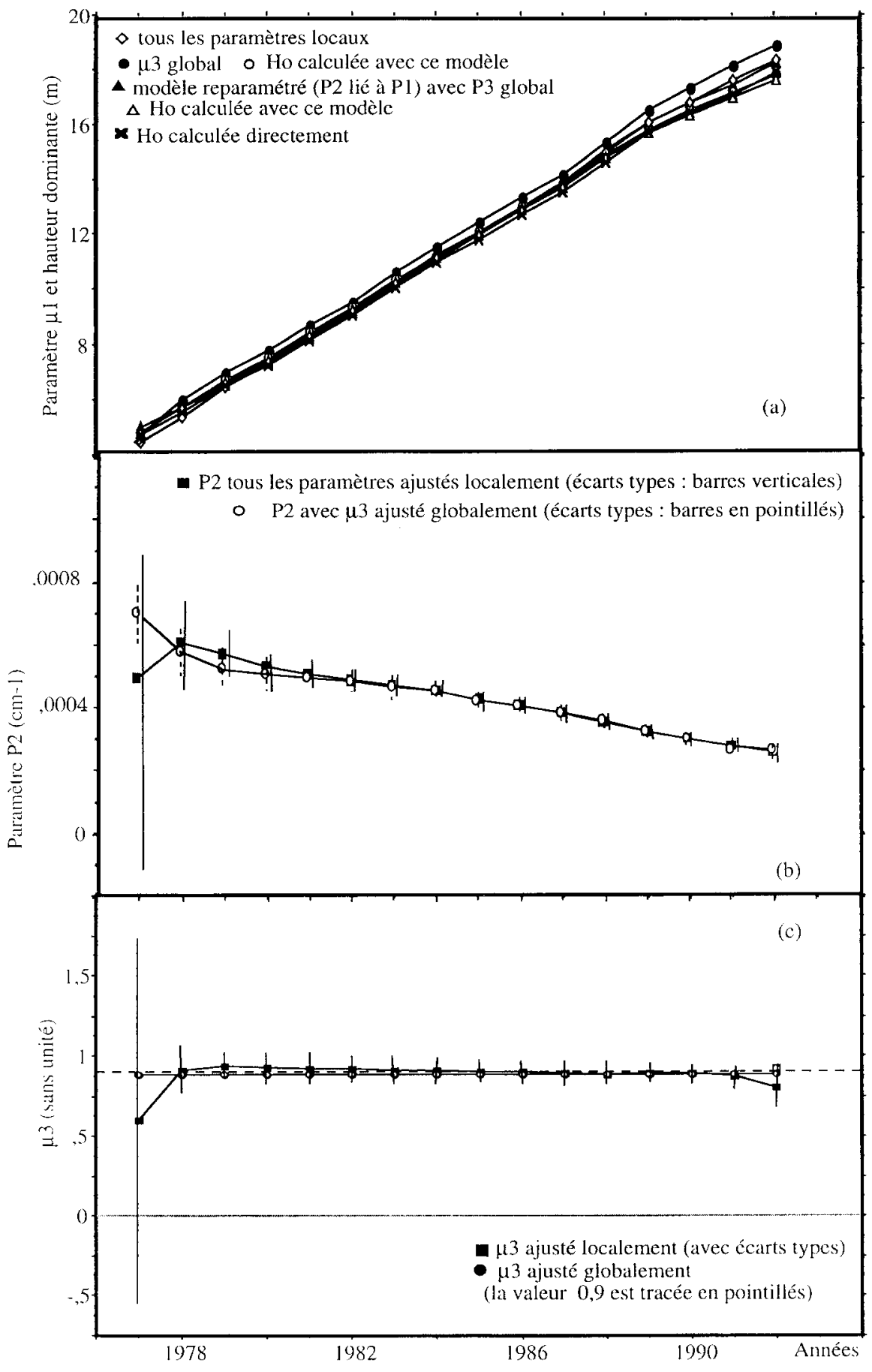


arbres en croissance libre (la classe N1 contenant trop peu d'arbres), pour lesquels le besoin d'une stabilité mécanique est assez fort et peut être plus limitant que la structuration sociale : on parle d'effet inhibiteur de la croissance libre sur la croissance en hauteur.

\section{CONCLUSION}

Pour conclure il est important de rappeler le premier résultat de cette étude : ce modèle, établi pour des feuillus, s'ajuste bien pour l'épicéa commun, ce qui élargit son domaine d'application. Ce résultat vient appuyer l'hypothèse sous-jacente du modèle supposé rendre compte de la différenciation sociale dans des peuplements où joue une forte compétition pour la lumière. Cette différenciation sociale se traduisait chez le hêtre et le chêne par l'existence d'une classe sociale en situation d'équilibre strict entre assimilation et maintenance (respiration et renouvellement d'organes vitaux) : la pente à l'origine était commune pour toutes les années et représentait des arbres du sous-étage dans cet état d'équilibre, sans croissance en hauteur ni en circonférence. Pour l'épicéa cet équilibre n'existe plus : la pente à l'origine augmente avec l'âge du peuplement. En effet les arbres les plus dominés utiliseront en quelque sorte leurs "dernières forces" (c'est à dire le peu d'énergie de leur assimilation) pour pousser un peu en hauteur et approcher de la lumière. En comparant un couvert forestier de hêtres relativement clair à un couvert d'épicéas le plus souvent opaque, une première interprétation de cette différence de comportement est que l'arrêt de la course pour la croissance en hauteur dans le peuplement d'épicéas signifie, à très brève échéance, l'arrêt de l'assimilation photosynthétique et la mort de l'arbre. II n'existe alors pas de sous-étage différencié. Cependant cette étude ne concerne qu'un peuplement jeune, pour lequel la remontée du couvert forestier est relativement rapide tandis que les travaux de Dhôte et de Hercé portaient sur des peuplements plus âgés (plus de 40 ans et généralement plus de 60 ans). Dans notre peuplement d'épicéas, la pente à l'origine de l'hyperbole pourrait se stabiliser avec le ralentissement de la croissance en hauteur. Cette dernière interprétation est appuyée par des récents travaux de Dhôte (résultats non publiés) : sur des peuplements plus jeunes de chênes (environ 30 ans) la pente à l'origine $\left(\mu_{2}\right)$ ne serait pas encore stabilisée (diminution de 0,6 à 0,35 ). Pour arbitrer entre ces deux facteurs (âge et essence), il conviendrait donc de travailler sur des peuplements plus âgés. Quel que soit le mécanisme sous-jacent, il est intéressant de constater que le modèle hyperbolique s'adapte dans les deux cas à la liaison hauteur-circonférence, seul le reparamétrage est changé.

Le deuxième résultat concerne le paramètre de forme $\left(\mu_{3}\right)$ qui est assez stable au cours du temps et peut même être fixé à 0,9 pour l'épicéa. Dhôte et de Hercé trouvaient des valeurs plus fortes $(0,98$ pour le hêtre et 0,99 pour le chêne), mais pour des

Fig 7. Évolutions des paramètres en fonction de l'âge (années en abscisse), dans le cas du modèle complet (tous les paramètres ajustés localement), du modèle avec $\mu_{3}$ global et du modèle reparamétré $\left(P_{2}\right.$ lié à $P_{1}$ ) avec $\mu_{3}$ global. a. Aux variations du paramètre $\mu_{1}$ on compare celles de la hauteur dominante (calculée avec la hauteur moyenne des 100 plus gros arbres à l'hectare ou à partir de la circonférence dominante et des modèles $(H O=f(C o))$; b Pour comparer le modèle complet au modèle reparamétré, on étudie les variations de $P_{2}$ (pour le modèle complet, $P_{2}=\mu_{2} / \mu_{1}$ ); c. Valeurs de $\mu_{3}$ avec la valeur de 0,9 tracée en pointillés. 
peuplements plus âgés. Pour des peuplements plus jeunes de chênes (environ 30 ans), le paramètre de forme serait plus faible ( 0,7 à 0,8 : Dhôte, communication personnelle). Pour des peuplements âgés (de plus de 100 ans) mais très clairs de hêtre ou de chêne, le paramètre de forme $\left(\mu_{3}\right)$ est aussi plus faible $(0,6$ à 0,8 : Dhôte, communication personnelle). Ce paramètre de forme pourrait ainsi être lié à l'état d'avancement de la fermeture du couvert. Cependant, la forme plus douce de l'hyperbole se traduit en pratique par une diminution plus rapide de la hauteur dominante, avec la densité, c'est-à-dire avec le décalage vers la gauche du nuage de points. Or, d'après Assmann (1970), l'épicéa est une essence particulièrement sensible à de très fortes densités pour sa croissance en hauteur dominante. Le faible paramètre de forme pourrait alors rendre compte aussi de la forte sensibilité de l'épicéa.

De ces deux premiers résultats, il apparaît en tout cas que les deux paramètres de forme et de pente à l'origine sont des indicateurs du niveau de compétition dans le peuplement et de l'arbitrage croissance en hauteur-croissance en circonférence qui en découle.

Le troisième résultat de cette étude est l'effet non-significatif de la densité sur le modèle. Ce résultat n'était pas évident a priori : si le modèle rend compte de la structuration sociale, elle-même sensible à la compétition, il aurait pu varier avec la densité. Le seul effet décelable est le déplacement de la distribution des données. En pratique ce résultat permet d'ajuster la relation hauteur-circonférence pour des peuplements ayant subi des traitements sylvicoles différents mais placés sur une même station.

Ce dernier résultat permet en particulier de dire que le modèle hyperbolique rend compte de l'invariance de la hauteur dominante pour une certaine gamme de densités. En revanche, si la structure du peuple- ment (relations interactives des arbres) n'est pas établie, ce modèle n'est sans doute pas valable, et en particulier pour des peuplements jeunes ou des arbres en croissance libre : la relation hauteur-circonférence pourrait alors être limitée en premier lieu par des contraintes de stabilité (MacMahon, 1976). En positionnant cette condition de stabilité, décrite par exemple par la relation allométrique $H=33,29^{*} D^{0,59}$ (Cusset ,1980), par rapport à notre faisceau de courbes, on remarque en effet qu'en peuplement l'ensemble des données s'éloigne largement de cette condition (fig 6b).

Finalement ces résultats soulignent l'intérêt de ce modèle hyperbolique : assez général pour être utilisé sur des feuillus ou des résineux, lié à la structuration sociale du peuplement, mais indépendant de la densité, et enfin possédant un paramètre très fortement corrélé à la hauteur dominante. II serait intéressant de poursuivre plus systématiquement son adaptation à d'autres essences sur une plus longue période de temps.

\section{REMERCIEMENTS}

Nous tenons à remercier $\mathrm{F}$ Colin et $\mathrm{F}$ Ningre pour toutes les informations qu'ils nous ont cordialement fournies concernant le dispositif clinal ; $G$ Thiriet et $P$ Garnier pour leur aide technique sur le terrain ; $R$ Canta qui nous a initiés aux mesures des cernes avec la machine Ecklund et enfin JF Dhôte, $P$ Dreyfus, J Bouchon et un lecteur anonyme pour leur commentaires sur le manuscrit de cet article.

\section{RÉFÉRENCES}

Assmann $E$ (1970) The principles of forest yield study. Pergamon Press, Oxford, $506 \mathrm{p}$

Bredenkamp BV (1991) Predicting tree height to supplement stand tables of Eucalyptus Grandis. South African For J 158, $3 \mathrm{p}$

Bolstad PV, Allen HL (1987) Height and diameter growth response in Loblolly pine stands following fertilisation. For Sci 33, 644-653 
Buford MA (1986) Height-diameter relationships at age 15 in Loblolly pine seed sources. For Sci 32, 812-818

Curtis RO (1967) Height-diameter and height-diameterage equations for second growth Douglas fir. For Sci $13,365-375$

Cusset G (1980) Sur des paramètres intervenant dans la croissance des arbres. La relation hauteur-diamètre de l'axe primaire aérien. Candollea 35, 231-255

Dhôte JF, de Hercé E (1994) Un nouveau modèle pour l'ajustement de faisceaux de courbes hauteur-diamètre. Can For Res (sous presse)

Décourt N (1970) Utilisation des dispositifs clinaux pour l'étude de la compétition dans les peuplements forestiers. Ve colloque d'écologie, 12-14 mars 1970, ENS, Paris

Dolph KL (1989) Height-diameter equations for younggrowth red fir in California and southern Oregon. Res Note PSW-408, Pacific Southern Forest and Range Experiment Station, Forest Service, USDA, $4 p$

Dreyfus $P(1990)$ Interaction génotype-densité et compétition dans un dispositif clinal d'épicéa commun. Ann Sci For 47, 1-16

Embry RS, Gottfried GJ (1971) Height-diameter equations for Arizona mixed conifers. USDA, For Ser Res Note, RM-191, 2 p

Farr WA, Demars DJ, Dealy JE (1989) Height and crown width related to diameter for open-growth western hemlock and Sitka spruce. Can For Res 19, 12031207

Ker JW, Smith JHG (1955) Advantages of the parabolic expression of height-diameter relationships. For Chron 236-246

Lanner RM (1985) On the insensitivity of height growth to spacing. For Eco Manage 13, 143-148
Larsen DR, Hann DW (1987) Height-diameter equations for 17 tree species in southern Oregon. Forest Research Laboratory, Oregon State University, Corvallis, $O R$, research paper $49,16 p$

MacMahon T (1973) Size and shape in biology. Elastic criteria impose limits on biological proportion and consequently on metabolic rates. Science 179, 1201-1204

Nys C, Bonischot R, Gelhaye D (1985) Réponse d'un peuplement de pin Laricio de Corse (Pinus nigra, espèce Laricio) à la fertilisation en Sologne. Rev For Fr 6, 482-486

Oswald $\mathrm{H}$ (1969) Conditions forestières et potentialités de l'épicéa en haute Ardèche. Ann Sci For 2, 562-567

Pardé J, Bouchon J (1988) Dendrométrie. Engref, Nancy, $328 \mathrm{p}$

Payandeh B (1984) Dimensional relationships for several tree species from the spruce-fir forest types of northwestern Ontario. Canadian Forestry Service Research Note, vol 4, $4 p$

Rai SN (1979) Diameter-height and diameter-girth relationships of some rain forest tree species of Karnataka (India). Malayan forester 42, 53-58

Seidel KW (1984) A western larch-Engelmann spruce spacing study in eastern Oregon: results after 10 years. USDA, Forest Service Note, PNW 409, $6 p$

Wang CH, Hann DW (1988) Height-diameter equations for 16 tree species in the central western Willamette valley of Oregon. Forest research Laboratory, Oregon State University, Corvallis, OR, research paper, vol 51, $7 \mathrm{p}$

Wykoff WR, Crookston CL, Stage AR (1982) User's guide to the stand prognosis model. USDA Forest Service, Intermountain Forest and Range Experiment Station, Ogden, Utah. General Technical Report INT-133, $122 p$ 